\title{
Engineering the optical properties of silicon using sub-wavelength structures
}

\author{
(Invited Paper) \\ R. Halir*, A. Ortega-Moñux*, A. Maese-Novo*, D. Pérez-Galacho*, L. Zavargo-Peche*, I. Molina-Fernández*, \\ J. G. Wangüemert Pérez* P. Cheben ${ }^{\dagger}$, J. H. Schmid ${ }^{\dagger}$, D.-X. Xu ${ }^{\dagger}$ S. Janz ${ }^{\dagger}$ and J.-M. Fédéli ${ }^{\ddagger}$ \\ ${ }^{*}$ Dpto. Ingeniería de Comunicaciones, ETSI Telecomunicación, Universidad de Málaga, 29010 Málaga, Spain \\ Email: robert.halir@ic.uma.es \\ ${ }^{\dagger}$ National Research Council of Canada, Ottawa, K1A 0R6, Canada \\ ${ }_{\ddagger}^{\ddagger}$ CEA, LETI, Minatec, Grenoble, France
}

\begin{abstract}
In most integrated optics platforms, including silicon-on-insulator, only minor modifications in refractive index are possible. The geometry of the waveguiding structure is thus the only degree of freedom for the design of devices. The use of sub-wavelength gratings (SWGs), i.e. structures that are small enough to suppress diffraction effects, enables local engineering of both refractive index and dispersion, thereby opening new possibilities for device design. Here we present some of the recent advances in refractive index and dispersion engineering using silicon SWGs, focussing on ultra-broadband and compact multimode interference couplers and directional couplers.
\end{abstract}

(C) 2013 IEEE, The 10th International Conference on Group IV Photonics

\section{INTRODUCTION}

A sub-wavelength grating (SWG) is, in its simplest form, a structure composed of alternating layers of two materials with a pitch substantially smaller than the wavelength of light propagating through it, so that diffraction effects are suppressed. The electromagnetic properties of such structures were first described more than half a century ago [1], predicting that they should essentially act as homogeneous materials with a refractive index lying between the indices of the materials that compose the grating. Silicon SWG waveguides were experimentally shown to provide a means to locally synthesize a wide range of effective indices in [2]. This concept of refractive index engineering with SWGs has widespread applications, including demultiplexers [3], waveguide crossings [4] and fiber-to-chip grating couplers [5]-[7].

Here we show how SWGs can be exploited to enhance the performance of well known integrated optical devices. We first present an ultra-short, high performance MMI realized with an index engineered SWG slot. We then introduce the notion of dispersion engineering, and apply it to an SWG assisted directional coupler achieving a fivefold bandwidth improvement compared to a conventional coupler, and an MMI coupler that covers a bandwidth of over $450 \mathrm{~nm}$.

\section{SHORTENING MMIS WITH INDEX ENGINEERING}

Figure 1(a) shows a SEM image of an MMI coupler with a sub-wavelength slot. As a conventional MMI this device is based on the self imaging principle, i.e. light launched into one the input waveguides (bottom of figure) excites multiple higher order modes in the central multimode region. As these modes propagate they interfere forming images of the input field that couple to the output waveguides. When properly designed, the SWG slot affects the propagation constants of the even modes in the multimode region such that the imaging distance is halved [8]. The pitch of the SWG and the size of holes is optimized with a specific Floquet mode simulation tool [9] resulting in a pitch of $\Lambda=238 \mathrm{~nm}$ and $50 \mathrm{~nm}$ holes. The optimum length of the multimode region is $\sim 23 \mu \mathrm{m}$, whereas a conventional MMI of the same width is $47 \mu \mathrm{m}$ long. As shown in Fig. 1(b) the measured extinction ratio of two devices in a back-to-back configuration is $15 \mathrm{~dB}$, indicating good device performance. In fact, a conventional MMI exhibits a slightly inferior extinction ratio, thereby confirming that the size reduction afforded by the SWG has no negative impact on device performance.

\section{DISPERSION ENGINEERING: DIRECTIONAL COUPLERS AND MMI COUPLERS}

The behavior of an SWG is generally wavelength dependent, as its effective index $\left(n_{\text {eff }}\right)$ increases sharply if the wavelength decreases sufficiently to approach the Bragg condition $\lambda_{\mathrm{Bragg}}=2 \Lambda n_{\text {eff }}$. Here we exploit this dependence to engineer the dispersion characteristics of optical devices, increasing their bandwidth.

Let us first consider the structure of the SWG assisted directional coupler shown in Fig. 2(a). The operation of this device is governed by the beat length of the even and odd supermodes in the coupling region: $L_{\pi}=\pi /\left(\beta_{\text {even }}-\beta_{\text {odd }}\right)$. In a conventional coupler $L_{\pi}$ increases with decreasing wavelength, because the propagation constant of the supermodes become increasingly similar. In the SWG assisted coupler this tendency is countered by the wavelength dependence of the SWG [10], so that $L_{\pi}$ 

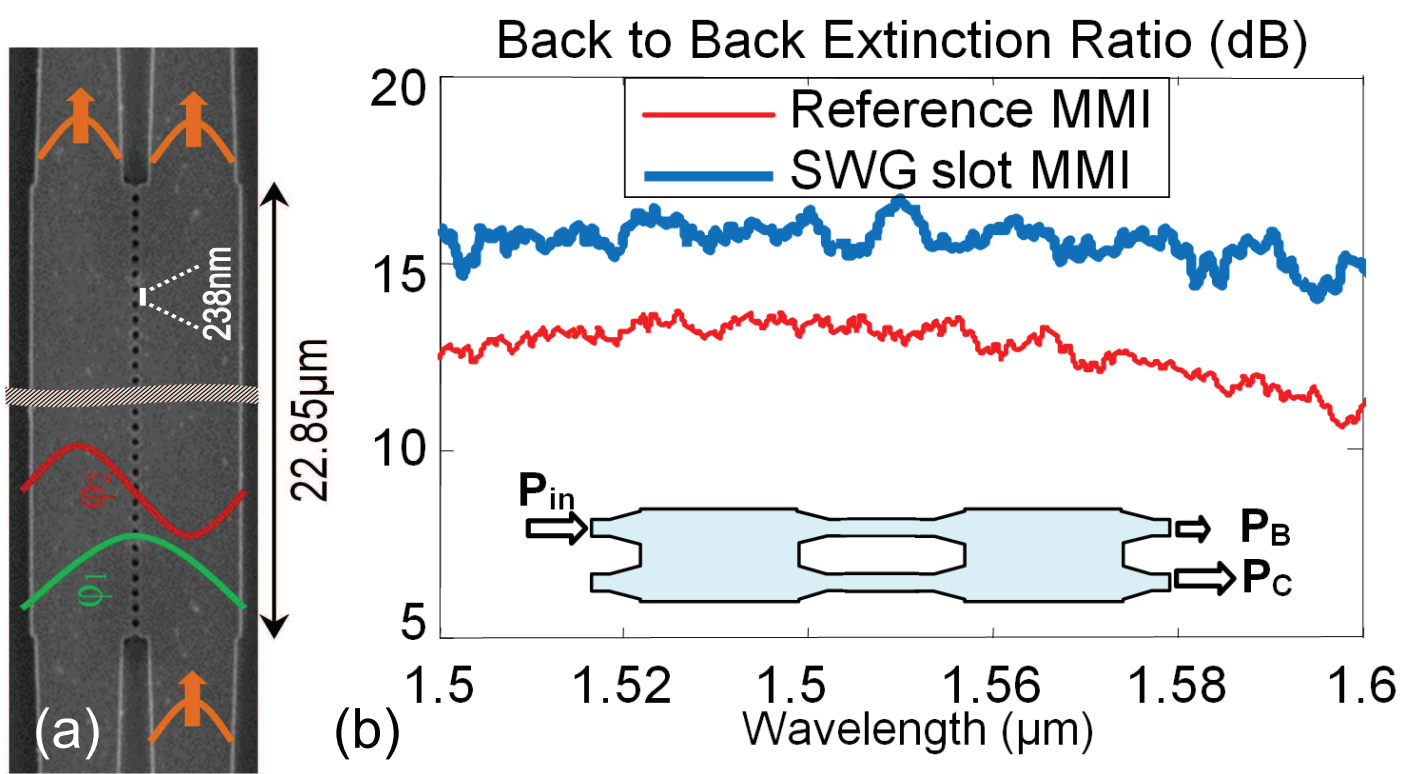

Fig. 1. (a) SEM image of an multimode interference with a sub-wavelength slot that reduces length in a factor of two compared to a conventional device. (b) Measured back-to-back extinction ratio of the slotted and the conventional device.
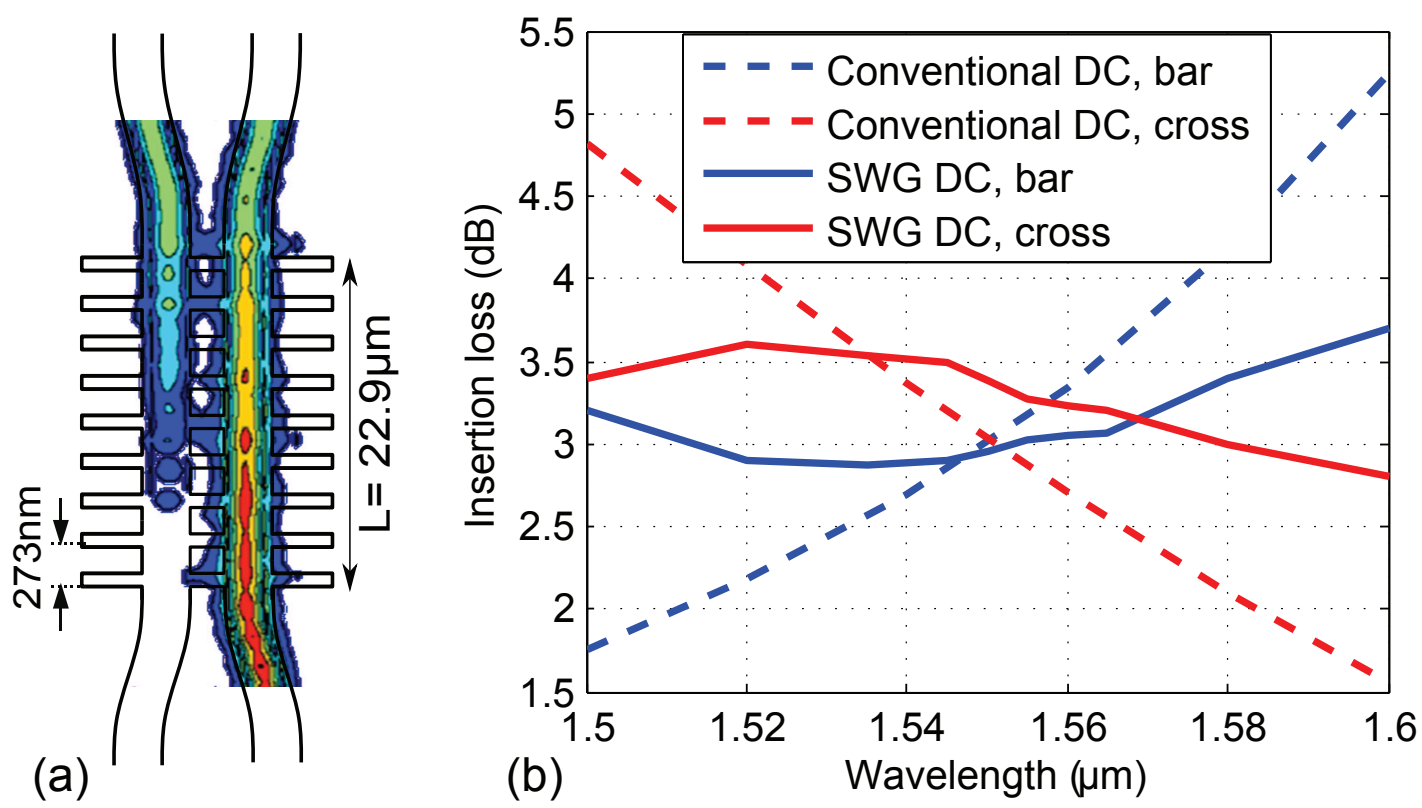

Fig. 2. (a) Directional coupler with an embedded SWG structure to increase the operational bandwidth - a simulation of the field propagation at $\lambda=1.55 \mu$ m is superimposed. (b) 3D-FDTD simulated insertion losses of the SWG assisted directional coupler and a conventional directional coupler.

is kept constant. In fact, as shown in Fig. 2(b) using SWG with a pitch of $238 \mathrm{~nm}$ the bandwidth of a conventional directional coupler that covers $\sim 20 \mathrm{~nm}$ is extended by a factor of five to $\sim 100 \mathrm{~nm}$.

Increasing the operational bandwidth in an MMI coupler requires control over the dispersion of several higher modes. This is achieved with the structure shown in Fig. 3(a). The SWG tapers at the device input and output control how many of the higher order modes are excited. Furthermore, they provide a smooth index transition from the silicon wire waveguides to the SWG multimode region [11]. Using our in-house software [9], the pitch of the SWG in the multimode region is adjusted to minimize the wavelength dependence of the distance at which the images of the input field form [12]. From Fig. 3(b) it is seen that this results in an operational bandwidth of more than $450 \mathrm{~nm}$, while a conventional MMI coupler only covers $\sim 100 \mathrm{~nm}$. 


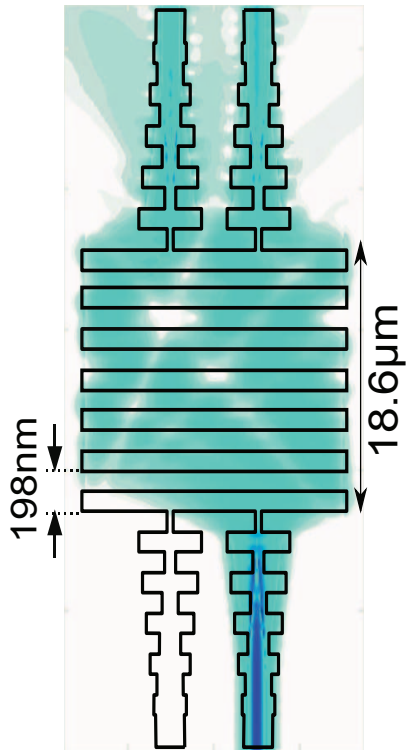

(a)

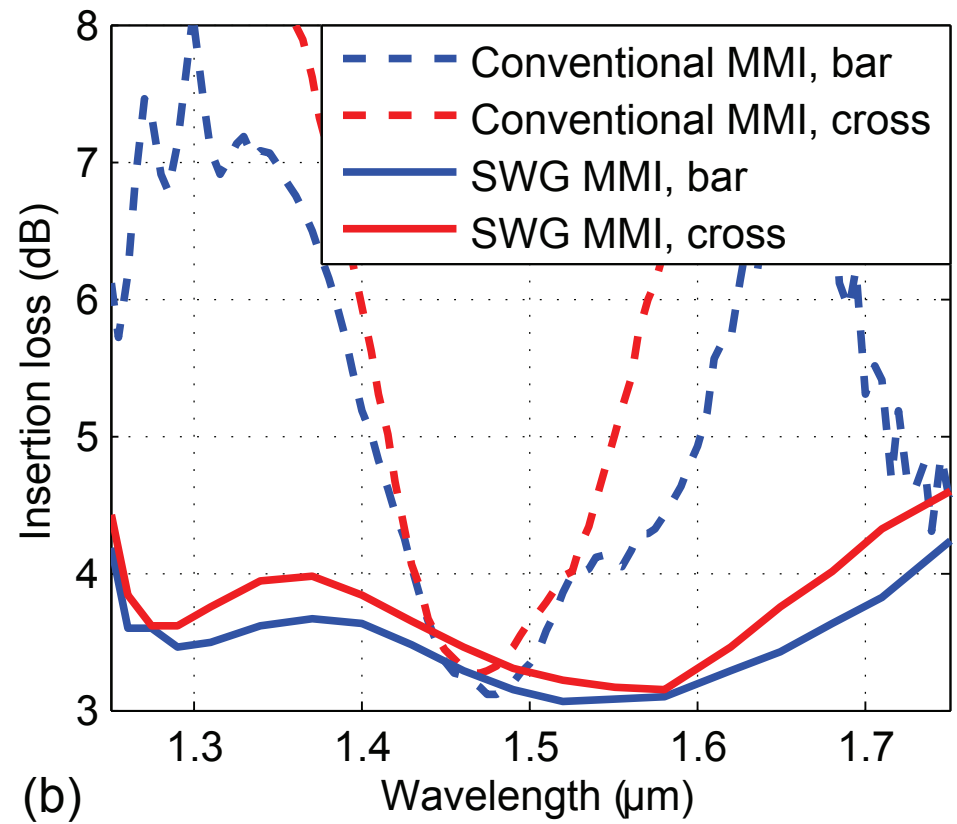

Fig. 3. (a) Dispersion engineered MMI coupler with SWG-tapered input and output waveguides - a simulation of the field propagation at $\lambda=1.47 \mu \mathrm{m}$ is superimposed. (b) 3D-FDTD simulated insertion losses of the dispersion engineered MMI coupler and a conventional MMI coupler.

\section{CONCLUSIONS}

We have shown that using silicon SWGs, the performance of coupling devices can be significantly improved, both in terms of size and bandwidth. This opens new opportunities for the design of ultra-broadband circuitry in silicon, as well as interesting prospect for reducing size and increasing bandwidth in other devices.

\section{ACKNOWLEDGEMENTS}

This work was supported by the Spanish Ministerio de Ciencia (project TEC2009-10152), the European Mirthe project (FP7-2010-257980), and the Universidad de Málaga - Campus de Excelencia Internacional Andalucía Tech.

\section{REFERENCES}

[1] S. M. Rytov, "The electromagnetic properties of finely layered medium," Sov. Phys. JETP, vol. 2, p. 466, 1956.

[2] P. Cheben, P. J. Bock, J. H. Schmid, J. Lapointe, S. Janz, D.-X. Xu, A. Densmore, A. Delâge, B. Lamontagne, , and T. J. Hall, "Refractive index engineering with subwavelength gratings for efficient microphotonic couplers and planar waveguide multiplexers," Opt. Lett., vol. 35, pp. 2526-2528, 2010.

[3] P. Bock, P. Cheben, A. Delâge, J. H. Schmid, D.-X. Xu, S. Janz, and T. J. Hall, "Demultiplexer with blazed waveguide sidewall grating and subwavelength grating structure," Opt. Express, vol. 16, pp. 17616-17625, 2008.

[4] P. J. Bock, P. Cheben, J. H. Schmid, J. Lapointe, A. Delâge, D.-X. Xu, S. Janz, A. Densmore, and T. J. Hall, "Subwavelength grating crossings for silicon wire waveguides," Opt. Express, vol. 18, no. 15, pp. 16146-16155, Jul 2010.

[5] R. Halir, P. Cheben, J. H. Schmid, R. Ma, D. Bedard, S. Janz, D.-X. Xu, A. Densmore, J. Lapointe, and I. Molina-Fernández, "Continuously apodized fiber-to-chip surface grating coupler with refractive index engineered subwavelength structure," Opt. Lett., vol. 35, no. 19, pp. 3243-3245, Oct 2010.

[6] R. Halir, L. Zavargo-Peche, D.-X. Xu, P. Cheben, R. Ma, J. Schmid, S. Janz, A. Densmore, A. Ortega-Monux, I. Molina-Fernandez, M. Fournier, and J.-M. Fedeli, "Single etch grating couplers for mass fabrication with duv lithography," Opt. Quantum Electron., pp. 1-6, invited.

[7] X. Chen, K. Xu, Z. Cheng, C. K. Fung, and H. K. Tsang, "Wideband subwavelength gratings for coupling between silicon-on-insulator waveguides and optical fibers," Opt. Lett., vol. 37, no. 17, pp. 3483-3485, 2012.

[8] A. Ortega-Moñux, C. Alonso-Ramos, A. Maese-Novo, R. Halir, L. Zavargo-Peche, D. Pérez-Galacho, I. Molina-Fernández, J. G. Wangüemert-Pérez, P. Cheben, J. H. Schmid et al., "An ultra-compact multimode interference coupler with a subwavelength grating slot," Laser \& Photon. Rev., vol. 7, pp. L12-L15, 2013.

[9] L. Zavargo-Peche, A. Ortega-Moñux, J. G. Wangüemert-Pérez, and I. Molina-Fernández, "Fourier based combined techniques to design novel sub-wavelength optical integrated devices," Prog. Electromagn. Res., vol. 123, pp. 447-465, 2012.

[10] R. Halir, A. Maese-Novo, A. Ortega-Moñux, I. Molina-Fernández, J. G. Wangüemert-Pérez, P. Cheben, D.-X. Xu, J. H. Schmid, and S. Janz, "Colorless directional coupler with dispersion engineered sub-wavelength structure," Opt. Express, vol. 20, no. 12, pp. 13 470-13477, Jun 2012.

[11] D. Pérez-Galacho, R. Halir, L. F. Zavargo-Peche, J. G. Wangüemert-Pérez, A. Ortega-Moñux, Íñigo Molina-Fernández, and P. Cheben, "Adiabatic transitions for sub-wavelength grating waveguides," in European Conference on Integrated Optics, 2012, paper 71.

[12] A. Maese-Novo, R. Halir, S. Romero-García, D. Pérez-Galacho, L. Zavargo-Peche, A. Ortega-Moñux, I. Molina-Fernández, J. G. Wangüemert-Pérez, and P. Cheben, "Wavelength independent multimode interference coupler," Opt. Express, vol. 21, no. 6, pp. 7033-7040, Mar 2013. 